\title{
Study on the law of disturbed coal seam permeability
}

\author{
JIANG Wan-gang ${ }^{1,2, a}$ \\ ${ }^{1}$ Gas Research Institute, China Coal Technology Engineering Group Chongqing Research \\ Institute, Chongqing 400037, China; \\ ${ }^{2}$ State Key Laboratory of Gas Disaster Detecting, Preventing and Emergency Controlling, \\ Chongqing 400037, China, \\ aiiangwangang@126.com
}

Keywords: mining coal; gas; permeability

Abstract. The 367 coal mining face in FengCheng ShangZhuang coal mine was selected for the project background, the law of the mining permeability is mirrored by the law of the flow of parallel hole that drain gas from virgin coal seam following by the moving of working face. According to the surveying data, the flow of hole had three sections, addition, declining, addition. The permeability of mining coal is mirrored indirect. The main reason was that the mining coal had experienced elastic deformation, plastic deformation, unloading expansion under the procession of coal mining, the permeability of mining coal is changed. It was important to study the law of gas transport, at the same time, it was the theory basis to control the gas effectively.

\section{Introduction}

According to the development of technology and equipment in coal mining continuously, advancing speed of work face has been increased soon, the seam is received compression and download of the abutment pressure in front of coal wall, so the permeability of the seam is changed, also the movement of gas in forward seam is changed. The study of movement of gas is important to control the problem of gas, and then the permeability of seam has close relationship with the law of gas movement, so the research of law of the permeability is the base. But at present, in the field of permeability, laboratory research is general, because the objective environment was changed, the size effect was ignored, and the effect of mining on permeability was not simulated in lab. So in this paper, the local monitor was used, the law of the changed permeability is got followed by the face advance, and at the same time, the basis is provided for controlling gas.

\section{The characteristic of seam in front of work face}

After the first ground pressure, followed by the face advance, the main roof captures periodically, it appears periodic pressure. so the seam's strength is varied by the face advance. According to the theory of rock pressure, there are three regions and three area upward and horizontal around the work face, the three rejoins and three area is shown in Fig.1[1].

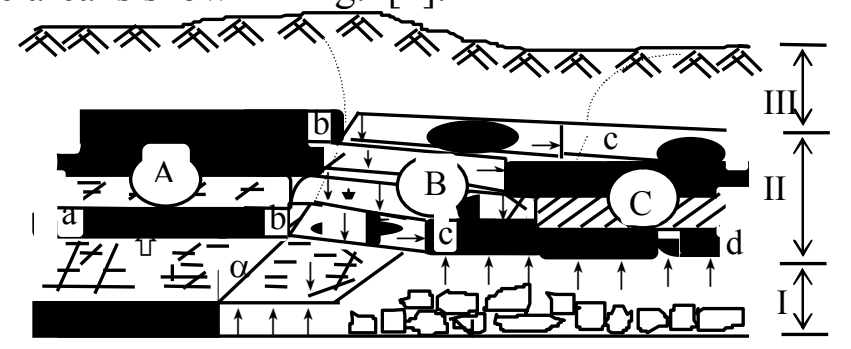

Fig.1 Three regions and three area

According to research the distributed law of the front abutment pressure, the law is got[2]: near by the wall of face, under the effect of main roof, the seam is broken, and it is unloaded, following by the abutment pressure conveying to the farther seam, the pressure is increased slowly, and to the eak , then 
going down to the virgin underground pressure. Following by the face advancing, the peak of abutment pressure advance, the area of abutment pressure is close to the wall in unloading condition, the seam is expanding, the macro fracture is increasing and breaking, the seam in the virgin underground pressure area is in the peak pressure area. The mining seam will be in the virgin pressure, abutment pressure, unloading condition, and advance.

\section{The permeability}

Permeability is the main parameter of seam, the permeable property is reacted directly in the seam. Research shows[3,4]: the flowing of gas in seam conforms the Dash law, from the Dash law, the permeability factor and permeability of seam are denoted:

$$
v=-p \cdot K
$$

Where $v$-the seepage velocity of gas, $\mathrm{m} / \mathrm{s} \Delta p$-pressure gradient of gas, $\mathrm{Pa} / \mathrm{m}, K$-permeability factor, $\mathrm{m} / \mathrm{s}$, the permeability factor turns on nature and geometric property, and it is related to the basic physical property of gas, in order to differentiate the influence between the characteristic of crack medium and nature of fluid, the permeability factor is written out like this[5]:

$$
k=K \cdot \mu
$$

Where $\mu$-coefficient of dynamic viscosity of gas, $10.26 \times 10^{-6} \mathrm{~m}^{2} / \mathrm{s}, k$-permeability, $10^{-4} \mu \mathrm{m}^{2}$, according to formula (1) jointing to formula (2), it is got that:

$$
k=-\frac{v \mu}{p}
$$

gas content of actual measurement is jointed to Langmuir equation, the gas pressure of this point is got, through determining the gas pressure around the hole continuously, then the gas pressure gradient is got. Langmuir equation:

$$
W=\frac{a b P}{1+b P} \cdot \frac{100-A_{d}-M_{a d}}{100} \cdot \frac{1}{1+0.31 M_{a d}} e^{n\left(t_{s}-t\right)}+\frac{F P}{10 \gamma}
$$

Where $W$-gas content of seam, $\mathrm{m}^{3} / \mathrm{t}, a, b$-constant of adsorption, $P$-absolute gas pressure, $\mathrm{MPa}, A_{d}$-ash of coal, \%, $M_{a d}$-moisture of coal,\%, F-porosity of coal,\%, $\gamma$-apparent density of coal, $\mathrm{t} / \mathrm{m}^{3}$, $t_{s}$-temperature of seam, $t_{s}=23{ }^{\circ} \mathrm{C}$, $t$-temperature of lab, $t=30{ }^{\circ} \mathrm{C}$. The gas pressure gradient $\Delta p$ is got around the hole $0.4575 \mathrm{MPa} / \mathrm{m}$.

\section{Local observation}

According to the site of ShangZhuang mine, the hole drainage volume was got by using the drainage hole in the seam at the haulage roadway in 367 face, each hole distance was $4 \mathrm{~m}$, the depth of hole was $65 \mathrm{~m}$, and the hole was numbered 1.2.3 ...according to the direction of face advance, the number 1 hole was $100 \mathrm{~m}$ distance to the coal wall, the definite arrangement of hole was Fig.2. Following by the face advance, the distance between the hole to coal wall, the concentration of hole drainage, negative pressure of drainage and drainage volume were recorded continuously, through analysis and comparison, the relationship between the concentration of hole drainage and the drainage volume to the advance of work face, combining with the gas pressure gradient, the gas permeability of mining coal around the hole was calculated. 


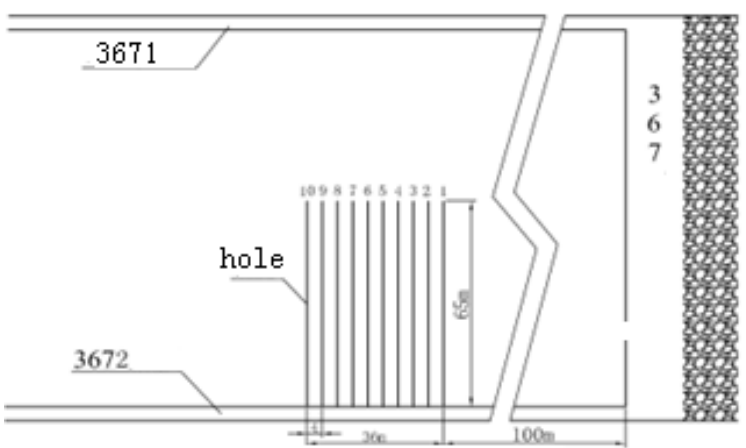

Fig.2 Arrangement of hole

\section{Confirm the permeability of seam}

According to the local monitor of the distance between hole and coal wall, the gas drainage volume of the hole (Tab.1), combining with the formula (3), the gas permeability of the disturbed coal seam in front of the work face was got. the Fig. 3 is the law between the permeability of mining coal seam and the distance to coal wall.

Tab.1 Data of drainage

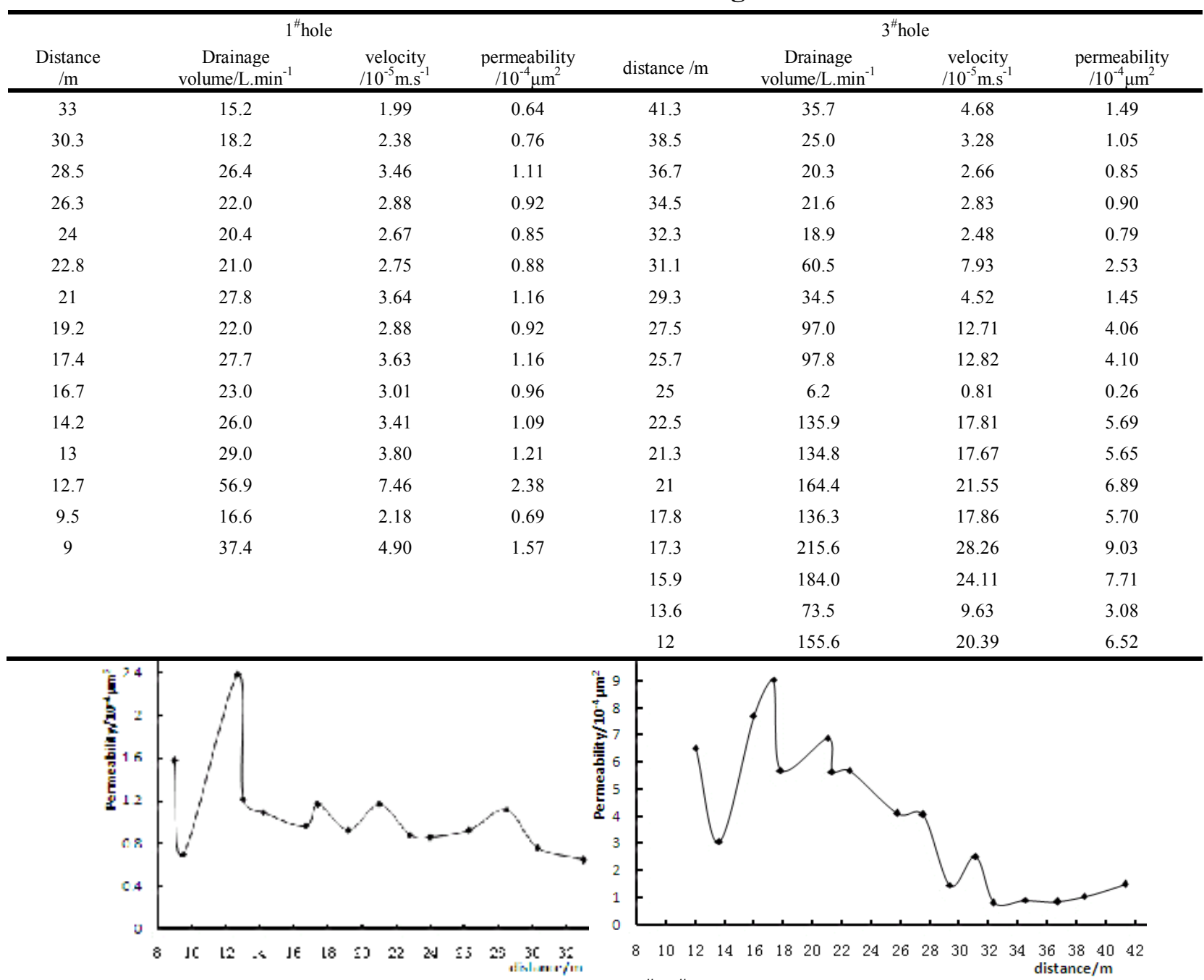

Fig.3 Relationship between distance of $1^{\#}, 3^{\#}$ hole to coal wall and permeability

It was found from the Fig.3, along with the diversification of the distance between coal wall and hole, the law of the permeability of mining coal seam in front of work face is following: around the coal wall, with the direction of advance, the permeability is going down from the peak, when the distance is $10 \mathrm{~m}$, the permeability rises rapidly, when it is $15 \mathrm{~m}$, the permeability achieves a peak again, the peak value strength is three times to the virgin seam permeability, then it declines slowly, and at last it recurs to 
original state. This phenomenon explain full, in mining beginning, the mining stress is not clear, the seam is spring condition, the former cracks enlarge continuous and the new cracks grow, so the permeability of seam improves slowly, when the face advances, the mining stress is strong gradually, then the seam is plastic condition, the cracks are close under the high stress, so the permeability also declines, the point that the abutment pressure is peak, is the lowest point of permeability, when the face continues to advance, the coal seam is unload condition, the crack grows and enlarges the macro-crack, the permeability increases and extends to the coal wall.

\section{Conclusions}

According to the continuous monitoring of drainage volume of hole, the gas flow velocity and gas pressure gradient etc. parameters were analyzed and calculated, then the permeability of mining seam is got.

On the basis of monitoring data, the permeability of mining seam in front of work face experience increase slowly, descending rapidly and rising fast, this is closely related to the condition of spring, plastic and unloading expansion under the abutment pressure continuous advance, it full explains that the mining effects the permeability obviously.

\section{References}

[1] Qian, Mingao, Shi,Pingwu. Rock Pressure and Strata Control[M].XuZhou: China University of Mining Press, 2003.

[2] Xie, Heping. Application of FLAC to predict ground surface displacements due to coal extraction and its comparative analysis, Chinese Journal of Rock Mechanics and Engineering, v18, n4, Aug, 1999, 397 401.

[3] Zhou, Shining.mechanism of the gas flowing in seam [J].J- ournal of China Coal Society, 1990, 15(1):61 67.

[4] Steve Z D H, Chu X Y, Xue F X. Dynamic nature of coal permeability of a longwall face [J].Int.J. Rock Mec.Min Set, 1999, 36(4):693 699.

[5] Deng, Zhigang, Qi, Qingxin, Li, Hongyan. Evolution law of mining coal permeability by real-time tacer monitoring system[J]. Journal of China Coal Society, 2008, 33(3):273 276. 